\title{
Interacting cosmic fluids and phase transitions under a holographic modeling for dark energy
}

\author{
Samuel Lepe ${ }^{1, a}$, Francisco Peña ${ }^{2, b}$ \\ ${ }^{1}$ Instituto de Física, Facultad de Ciencias, Pontificia Universidad Católica de Valparaíso, Avenida Brasil, 4950 Valparaiso, Chile \\ 2 Departamento de Ciencias Físicas, Facultad de Ingeniería y Ciencias, Universidad de La Frontera, Avenida Francisco Salazar, Casilla 54-D, \\ 01145 Temuco, Chile
}

Received: 14 December 2015 / Accepted: 5 September 2016 / Published online: 16 September 2016

(C) The Author(s) 2016. This article is published with open access at Springerlink.com

\begin{abstract}
We discuss the consequences of possible sign changes of the $Q$-function which measures the transfer of energy between dark energy and dark matter. We investigate this scenario from a holographic perspective by modeling dark energy by a linear parametrization and CPLparametrization of the equation of state $(\omega)$. By imposing the strong constraint of the second law of thermodynamics, we show that the change of sign for $Q$, due to the cosmic evolution, imply changes in the temperatures of dark energy and dark matter. We also discuss the phase transitions, in the past and future, experienced by dark energy and dark matter (or, equivalently, the sign changes of their heat capacities).
\end{abstract}

\section{Introduction}

Certain observational evidence seems to indicate that dark energy and dark matter, considered as dominant components of the cosmic fluid, can interact [1-4]. Perhaps an interaction between dynamical vacuum energy and matter is also possible [5] or a scenario where a particle creation mechanism which can lead to cosmic acceleration [6]. It appears unnatural to think that cosmic fluids coexist and do not interact with each other as is usual in the standard $\triangle \mathrm{CDM}$ model (both components evolve independently and satisfy the usual energy conservation equations). As is well known, $\triangle \mathrm{CDM}$, despite its success, is theoretically unappealing because of several well-known shortcomings. Motivated by that, new challenges emerge [7] as, for instance, to understand the nature of the dark energy and the nature of the phantom dark energy (not ruled out by observations), an interesting issue

\footnotetext{
${ }^{a}$ e-mail: samuel.lepe@ pucv.cl

b e-mail: francisco.pena@ufrontera.cl
}

which seems very difficult to understand within the $\Lambda \mathrm{CDM}$ framework.

On the other hand, dark energy and dark matter, both seen as ordinary fluids (perfect fluids), lead to a consistent and simple way to describe them. In other words, here, dark energy and dark matter are described under the hypothesis of perfect fluids and in the literature we find nothing against this assumption. In any case, it is an interesting approach if we are thinking that a perfect fluid is something that we know how to handle.

Components with interaction lead to a new perspective for describing/visualizing the cosmic evolution. There is a rich literature on the subject and for describing that interaction, for instance, various Ansatzes for $Q$ (defined below) are used. This is a first approximation to describe the interaction required by the observational data, but there is no formalism that allows us to obtain $Q$ from first principles.

In this work, we analyze thermodynamical aspects from the aforementioned interaction in the framework of two interacting fluids. As already said, we will use the holographic principle for modeling the dark energy where the emphasis will appear from the temperature, during the cosmic evolution, from each fluid (dark energy and dark matter. Phase transitions (seen as sign changes in the heat capacity) will also be discussed. $8 \pi G=c=1$ units will be used.

The organization of the paper is as follows: in Sect. 2 we present a brief description of two non-interacting fluids and we revise the behavior of its temperatures through the cosmic evolution. In Sect. 3 we incorporate interaction and we revise the involved thermodynamics bearing in mind the second law. In Sect. 4 we discuss the interaction under holographic considerations and we show the presence of phase transitions (sign changes in its heat capacities) experienced by both fluids during the evolution. In Sect. 5, we discuss the behavior of the Hubble parameter and cosmological evolution model. 
Finally, Sect. 6 is devoted to our conclusions. We have added an appendix to show explicitly the sign changes in the heat capacities of the interacting fluids.

\section{Non-interacting fluids and thermodynamics}

We consider, in the framework of General Relativity (GR), the non-interacting flat-FLRW scheme between two components,

$3 H^{2}=\rho_{\mathrm{de}}+\rho_{\mathrm{dm}}$

and

$$
\begin{array}{r}
\dot{\rho}_{\mathrm{de}}+3 H\left(1+\omega_{\mathrm{de}}\right) \rho_{\mathrm{de}}=0, \\
\dot{\rho}_{\mathrm{dm}}+3 H\left(1+\omega_{\mathrm{dm}}\right) \rho_{\mathrm{dm}}=0,
\end{array}
$$

where $\rho_{\mathrm{de}}$ denotes the dark energy density, $\rho_{\mathrm{dm}}$ denotes the dark matter density, $H$ is the Hubble parameter, and the dot denotes derivative with respect to the cosmic time. We assume that both components can be amenable to study by using a description under the rigorous scope of the thermodynamic laws. Then, by using the equation $T \mathrm{~d} S=$ $\mathrm{d}(\rho V)+p \mathrm{~d} V$, for a generic fluid, where $T$ is the temperature, $S$ the entropy, $\rho$ the energy density, $p$ its pressure, and $V$ the physical volume, besides the integrability condition $\partial^{2} S / \partial T \partial V=\partial^{2} S / \partial V \partial T$, we can obtain the following equation for the temperature during the cosmic evolution [810]:

$$
\begin{aligned}
\frac{\mathrm{d} T}{T} & =-\frac{\mathrm{d} V}{V}\left(\frac{\partial p}{\partial \rho}\right)_{V}=-3 H \mathrm{~d} t\left(\frac{\partial p}{\partial \rho}\right)_{a}, \\
& \rightarrow T(z)=T(0) \exp \left(3 \int \frac{\mathrm{d} z}{1+z} \omega(z)\right),
\end{aligned}
$$

where we have used $p=\omega \rho, 1+z=a_{0} / a$ with $z$ being the redshift parameter and $a$ the cosmic scale factor with $a_{0}=a(0)$. Since $V=\Omega_{0} a^{3}$ (for a spatially flat section $\Omega_{0}=4 \pi / 3$, see [11-13]), we obtain $\mathrm{d} V / V=3 \mathrm{~d} a / a=$ $3 H \mathrm{~d} t$. By considering for dark energy $\omega_{\mathrm{de}}(z) \approx-1$ and for non-relativistic dark matter $p_{\mathrm{dm}}=n T_{\mathrm{dm}}, n$ being the number density and $\rho_{\mathrm{dm}}=n m+3 n T / 2$ with $m$ the rest mass, where $T_{\mathrm{dm}} \ll m$ [8], we can obtain $T_{\mathrm{dm}}(z) \sim(1+z)^{2}$ after using (3). So,

$$
T_{\mathrm{de}}(z)=T_{\mathrm{de}}(0)(1+z)^{-3}, \quad T_{\mathrm{dm}}(z)=T_{\mathrm{dm}}(0)(1+z)^{2},
$$

i.e., $T_{\mathrm{de}}(z)$ grows and $T_{\mathrm{dm}}(z)$ decreases when $z$ goes to the future. This fact appears to be unusual if we want to think in energy transference from dark energy to dark matter, at least from some $z_{e}$ in the past. At equilibrium, we have

$$
\begin{aligned}
& T_{\mathrm{de}}\left(z_{e}\right)=T_{\mathrm{dm}}\left(z_{e}\right), \\
& \Longrightarrow z_{e}=\left[T_{\mathrm{de}}(0) / T_{\mathrm{dm}}(0)\right]^{1 / 5}-1, \\
& \rightarrow\left\{\begin{array}{c}
z_{e} \in \text { past } \Longrightarrow T_{\mathrm{de}}(0) / T_{\mathrm{dm}}(0)>1, \\
z_{e} \in \text { future } \Longrightarrow T_{\mathrm{de}}(0) / T_{\mathrm{dm}}(0)<1 .
\end{array}\right.
\end{aligned}
$$

Nowadays we would expect that $T_{\mathrm{de}}(0)>T_{\mathrm{dm}}(0)$ (see below) and in this case $z_{e}$ belongs to the past. But the problem arises when $z_{e}$ is in the future. If we do not have equilibrium through the evolution, we can think in two options: only one sign of $Q$ and then only one option for the inequality between the temperatures, $T_{\mathrm{de}}(z)>T_{\mathrm{dm}}(z)$ or $T_{\mathrm{de}}(z)<T_{\mathrm{dm}}(z)$. Nevertheless, there are holographic arguments in order to justify, at least on a theoretical level, both options for the sign of $Q$ [14]. As a second option we can imagine that even when there are sign changes in $Q$, the amount of energy transferred is not enough for generating changes in the relation between $T_{\mathrm{de}}(z)$ and $T_{\mathrm{dm}}(z)$. But the thermodynamical constraint given by the second law rejects the latter option.

By following Ref. [8], we ascribe a temperature (Gibbs integrability condition) to the dark energy in the form $T_{\mathrm{de}} \sim$ $\rho_{\mathrm{de}}^{\omega /(\omega+1)}$, and we see that if $-1<\omega<0, T_{\mathrm{de}}$ increases when $\rho_{\text {de }}$ decreases and vice versa. If we assume that the given relationship between temperature and energy density is valid for $\omega<-1$ (phantom dark energy, not ruled out by the observational data), $T_{\mathrm{de}}$ and $\rho_{\mathrm{de}}$, both increase as time evolves and dark energy increasing in the future appears to be not only a conjecture.

On the other hand, if we write for dark matter $T_{\mathrm{dm}} \sim$ $\rho_{\mathrm{dm}}^{\omega /(\omega+1)}$, we can see that $\omega=0$ (dust) leads to $T_{\mathrm{dm}}=\mathrm{const}$ and if this were the case, we would have, from a thermodynamical point of view, a fluid without the ability of interacting with others. Strictly speaking, the limiting case of dust has null temperature and then we have the same, that is, a fluid without the ability of interacting with others. Roughly speaking, when we consider a pressureless fluid we are thinking in $p \approx 0$, i.e., non-null pressure or $\omega \approx 0$ and also non-null temperature for it. In this case, whether we can have a fluid capable of interacting with others.

In the next sections we introduce the interaction and discuss its consequences for the evolution of the temperatures.

\section{Interacting fluids and thermodynamics}

By using the interacting scheme

$$
\begin{gathered}
\dot{\rho}_{\mathrm{de}}+3 H\left(1+\omega_{\mathrm{de}}\right) \rho_{\mathrm{de}}=-Q, \\
\dot{\rho}_{\mathrm{dm}}+3 H\left(1+\omega_{\mathrm{dm}}\right) \rho_{\mathrm{dm}}=Q,
\end{gathered}
$$

or, equivalently

$$
\begin{gathered}
\dot{\rho}_{\mathrm{de}}+3 H\left(1+\omega_{\mathrm{de}}^{\mathrm{eff}}\right) \rho_{\mathrm{de}}=0, \\
\dot{\rho}_{\mathrm{dm}}+3 H\left(1+\omega_{\mathrm{dm}}^{e f f}\right) \rho_{\mathrm{dm}}=0,
\end{gathered}
$$


where

$\omega_{\mathrm{de}}^{\mathrm{eff}}=\omega_{\mathrm{de}}+\frac{Q}{3 H \rho_{\mathrm{de}}}$ and $\omega_{\mathrm{dm}}^{\mathrm{eff}}=\omega_{\mathrm{dm}}-\frac{Q}{3 H \rho_{\mathrm{dm}}}$,

so that according to (3) the temperatures are given, respectively, by

$$
\begin{aligned}
& T_{\mathrm{de}}(z) \sim \exp \left(3 \int \frac{\mathrm{d} z}{1+z} \omega_{\mathrm{de}}^{\text {eff }}\right)=\exp \left(3 \int \frac{\mathrm{d} z}{1+z} \omega_{\mathrm{de}}\right) \\
& \quad \times \exp \left(3 \int \frac{\mathrm{d} z}{1+z} \frac{Q}{H \rho_{\mathrm{de}}}\right)
\end{aligned}
$$

and

$$
\begin{aligned}
& T_{\mathrm{dm}}(z) \sim \exp \left(3 \int \frac{\mathrm{d} z}{1+z} \omega_{\mathrm{dm}}^{\mathrm{eff}}\right)=\exp \left(3 \int \frac{\mathrm{d} z}{1+z} \omega_{\mathrm{dm}}\right) \\
& \quad \times \exp \left(-3 \int \frac{\mathrm{d} z}{1+z} \frac{Q}{H \rho_{\mathrm{dm}}}\right) .
\end{aligned}
$$

Here we have considered a generic $\omega_{\mathrm{dm}}$ for dark matter without any special commitment with it. In Sect. 4 we will use $\omega_{\mathrm{dm}}=0$ (dust).

On the other hand, in the presence of interaction, the entropy production associated to the interaction is [15]

$\forall t, \quad \dot{S}_{\mathrm{de}}(t)+\dot{S}_{\mathrm{dm}}(t)=\left(\frac{Q(t)}{T_{\mathrm{dm}}(t)}+\frac{(-Q(t))}{T_{\mathrm{de}}(t)}\right) \geqslant 0$,

or, by using the redshift parameter

$$
\begin{aligned}
& \forall z, \quad-(1+z) H(z) \frac{\mathrm{d}}{\mathrm{d} z}\left[S_{\mathrm{de}}(z)+S_{\mathrm{dm}}(z)\right] \\
& =\left(\frac{1}{T_{\mathrm{dm}}(z)}-\frac{1}{T_{\mathrm{de}}(z)}\right) Q(z) \geqslant 0
\end{aligned}
$$

i.e. $T_{\mathrm{de}}(z)>T_{\mathrm{dm}}(z)$ at late times $\Longrightarrow Q(z)>0$. However, at early times $T_{\mathrm{de}}(z)<T_{\mathrm{dm}}(z)$ (a reasonable assumption), meaning that we should have $Q(z)<0$ in order to satisfy the second law. As was said before, it may seem strange that today $T_{\mathrm{de}}(z)$ grows with $z$ if dark energy is transferring energy to dark matter $(Q(z)>0)$ at least from some $z_{e}(5)$. Can we have a signal of a negative heat capacity for dark energy? As we will see in the next section, the incorporation of interaction explicitly shows this fact. Moreover, the dark matter shows also phase transitions, in the past and in the future. Sign changes of $Q$ (one in the past and another in the future) can be visualized in Ref. [14] where a holographic modeling for the dark energy was used with the linear parametrization $\omega_{\mathrm{de}}(z)=\omega_{\mathrm{de}}(0)+\sigma z$, and $\sigma=\mathrm{const}$ [16].

We end this section by making a consistency check. By using the usual concepts of thermodynamics, we deduce the equation for the evolution of the temperature for a generic fluid denoted by $\rho$. We start by setting $\rho=\rho(V, T)$ and $p=p(V, T)$, a reasonable setup. So, $\dot{\rho}=a H\left(\frac{\partial \rho}{\partial a}\right)_{T}+\left(\frac{\partial \rho}{\partial T}\right)_{a} \dot{T}$,

and $V=(4 \pi / 3) a^{3}$ so that $\dot{V}=3 V H$ and $(\partial \rho / \partial V)_{T}=$ $(a / 3 V)(\partial \rho / \partial a)_{T}$. On the other hand, from the second law with the integrability condition given before, it is straightforward to obtain the expression

$$
\left(\frac{\partial \rho}{\partial a}\right)_{T}=\frac{3 T}{a}\left[\left(\frac{\partial p}{\partial T}\right)_{a}-\frac{p+\rho}{T}\right] .
$$

By replacing this last expression in (13) and by using the non-conservation equation

$\dot{\rho}=Q_{f}-3 H(\rho+p)$,

where $Q_{f}=-Q$ for dark energy and $Q_{f}=Q$ for dark matter, we can obtain

$\frac{\dot{T}}{T}=-3 H\left(\frac{\partial p}{\partial \rho}\right)_{a}+\left(\frac{\partial \rho}{\partial T}\right)_{a}^{-1} \frac{Q_{f}}{T}$,

and by using the redshift parameter, we write the last equation in the form

$\frac{\mathrm{d} T}{T}=\frac{\mathrm{d} z}{1+z}\left[3\left(\frac{\partial p}{\partial \rho}\right)_{z}-\frac{Q_{f}}{H}\left(\frac{\partial \rho}{\partial T}\right)_{z}^{-1} \frac{1}{T}\right]$,

so that, for dark energy,

$\frac{\mathrm{d} T_{\mathrm{de}}}{T_{\mathrm{de}}}=\frac{\mathrm{d} z}{1+z}\left[3\left(\frac{\partial p_{\mathrm{de}}}{\partial \rho_{\mathrm{de}}}\right)_{z}+\frac{Q}{H}\left(\frac{\partial \rho_{\mathrm{de}}}{\partial T_{\mathrm{de}}}\right)_{z}^{-1} \frac{1}{T_{\mathrm{de}}}\right]$,

and for dark matter

$\frac{\mathrm{d} T_{\mathrm{dm}}}{T_{\mathrm{dm}}}=\frac{\mathrm{d} z}{1+z}\left[3\left(\frac{\partial p_{\mathrm{dm}}}{\partial \rho_{\mathrm{dm}}}\right)_{z}-\frac{Q}{H}\left(\frac{\partial \rho_{\mathrm{dm}}}{\partial T_{\mathrm{dm}}}\right)_{z}^{-1} \frac{1}{T_{\mathrm{dm}}}\right]$.

Now, we compare the expressions given in (9) and (18), i.e.,

$\frac{\mathrm{d} T_{\mathrm{de}}}{T_{\mathrm{de}}}=\frac{\mathrm{d} z}{1+z}\left[3 \omega_{\mathrm{de}}(z)+\frac{Q}{H} \frac{1}{\rho_{\mathrm{de}}}\right]$

and

$\frac{\mathrm{d} T_{\mathrm{de}}}{T_{\mathrm{de}}}=\frac{\mathrm{d} z}{1+z}\left[3 \omega_{\mathrm{de}}(z)+\frac{Q}{H}\left(\frac{\partial \rho_{\mathrm{de}}}{\partial T_{\mathrm{de}}}\right)_{z}^{-1} \frac{1}{T_{\mathrm{de}}}\right]$,

where $\left(\partial p_{\text {de }} / \partial \rho_{\text {de }}\right)_{z}=\omega_{\text {de }}(z)$. The consistency between (20) and (21) indicates that

$\rho_{\mathrm{de}}=\left(\frac{\partial \rho_{\mathrm{de}}}{\partial T_{\mathrm{de}}}\right)_{z} T_{\mathrm{de}}$, 
and the same occurs if we compare (10) and (19)

$\rho_{\mathrm{dm}}=\left(\frac{\partial \rho_{\mathrm{dm}}}{\partial T_{\mathrm{dm}}}\right)_{z} T_{\mathrm{dm}}$

as expected (see [17]).

\section{Sign change of $Q$ and holography}

One approach for treating the $Q$-function is by considering the following Ansatz: $Q=3 H\left(\lambda_{1} \rho_{\mathrm{de}}+\lambda_{2} \rho_{\mathrm{dm}}\right)$, where $\lambda_{1}$ and $\lambda_{2}$ are both constant parameters to be adjusted by observations. According to observational settings, the two parameters have equal sign and so there is no sign change in $Q$ $[18,19]$.

The second approach, which we will use from now on, is based on a holographic model

$\rho_{\mathrm{de}}(z)=3 H^{2}(z)\left[\alpha-\frac{\beta}{2}(1+z) \frac{\mathrm{d} \ln H^{2}(z)}{\mathrm{d} z}\right]$,

where $\alpha$ and $\beta$ are both positive parameters which are well confined by the observational data: $\beta<\alpha<1$ [14,20,21].

The infrared cut-off given for $\rho_{\mathrm{de}}[20,21]$ can be understood as a generalization of the model $\rho_{\mathrm{de}} \sim-R$ [22], where $R$ is the Ricci scalar given by $R=-6\left(2 H^{2}+\dot{H}+k / a^{2}\right)$ or as an extension of the holographic model $\rho_{\mathrm{de}}=3 \alpha \mathrm{H}^{2}$ proposed by $\mathrm{Li}$ [23]. In the last case, the key idea is to use the holographic principle [24] and its possible role in cosmology. This approach is an open issue and, in this philosophy, the model given in (24) is an interesting starting point in order to visualize what we mean by dark energy. This is a crucial fact if we are thinking (as usual) of dark energy as a cosmological constant, although the observational data would indicate that $\rho_{\mathrm{de}}$ is not necessarily a constant [25]. In this sense, the $\Lambda \mathrm{CDM}$ model could be questioned, despite their successes.

By using (24) besides (1) and (6), it is possible to write

$\rho_{\mathrm{de}}(z)=3 H^{2}(z)\left(\frac{2 \alpha-3 \beta}{2+3 \beta \omega_{\mathrm{de}}(z)}\right)$,

and so

$\rho_{\mathrm{dm}}(z)=3 H^{2}(z)\left(\frac{2(1-\alpha)+3 \beta\left(1+\omega_{\mathrm{de}}(z)\right)}{2+3 \beta \omega_{\mathrm{de}}(z)}\right)$,

so that we can obtain an explicit expression for $Q$

$$
\begin{aligned}
& \frac{Q}{9 H^{3}}(z)=-(2 \alpha-3 \beta) \\
& \quad \times \frac{\left[2(1-\alpha)+3 \beta\left(1+\omega_{\mathrm{de}}\right)\right] \omega_{\mathrm{de}}+\beta(1+z) \mathrm{d} \omega_{\mathrm{de}} / \mathrm{d} z}{\left(2+3 \beta \omega_{\mathrm{de}}\right)^{2}} .
\end{aligned}
$$

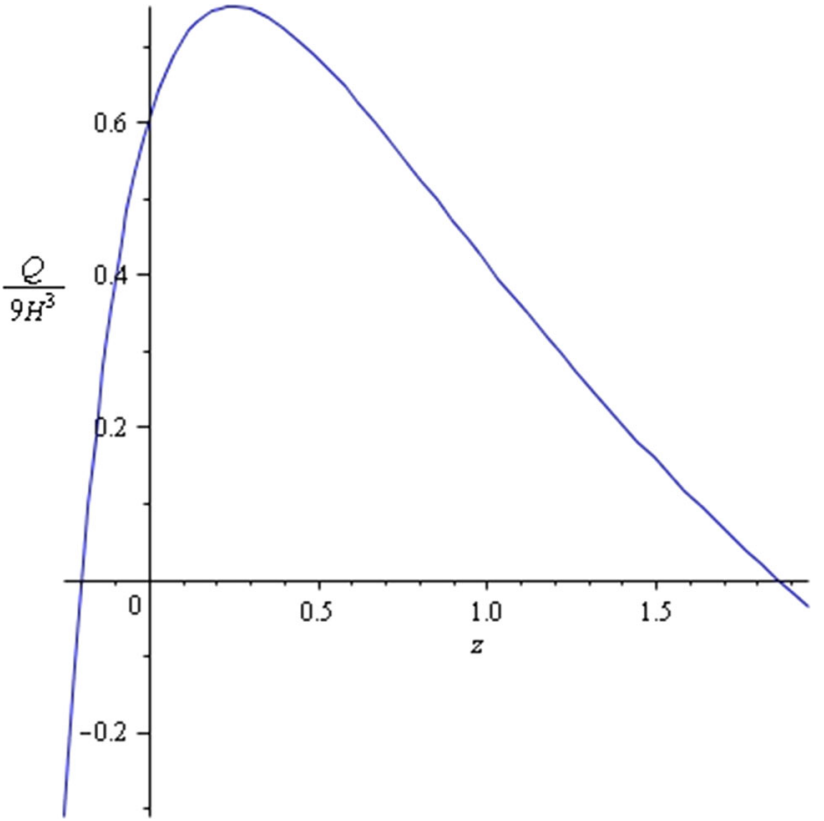

Fig. 1 The behavior of $Q$ and its two sign changes: for $-0.2<z<$ 1.86 we have $Q>0$, and $Q<0$ otherwise

The interaction sign can change through the evolution as can be seen from the Ansatz $\omega_{\mathrm{de}}(z)=\omega_{\mathrm{de}}(0)+\sigma z$. Therefore, according to (27) and by using the Ansatz given before, $Q(z)$ experiences two sign changes (Fig. 1), one in the past and another in the future, as can be found in [14]. But, what means a sign change of $Q$ at late times?, would we have dark matter phase again?, the current accelerated expansion would be transient previous to a possible future collapse? [26].

On the other hand, and under an entropic philosophy (entropic cosmology) in which we have an amount of nonconservation energy, we can observe sign changes in it and, in particular, that sign is mainly dependent on the equation of state $(\omega)$ in each stage of the cosmic evolution [27].

Assuming a holographic principle, also, if we are considering an interaction between the bulk and the boundary of the space-time, we can see sign changes in $Q(z)$ [28].

Now, by using (27) together with (26) and (25) in (8), we write

$\omega_{\mathrm{de}}^{\mathrm{eff}}=\left(\frac{2 \alpha-3 \beta}{2+3 \beta \omega_{\mathrm{de}}}\right)\left[\omega_{\mathrm{de}}-\frac{\beta(1+z)}{2 \alpha-3 \beta} \frac{\mathrm{d} \omega_{\mathrm{de}}}{\mathrm{d} z}\right]$

and

$$
\begin{aligned}
& \omega_{\mathrm{dm}}^{\mathrm{eff}}=\omega_{\mathrm{dm}}+\left(\frac{2 \alpha-3 \beta}{2+3 \beta \omega_{\mathrm{de}}}\right) \\
& \quad \times\left[\omega_{\mathrm{de}}+\frac{\beta(1+z)}{2(1-\alpha)+3 \beta\left(1+\omega_{\mathrm{de}}\right)} \frac{d \omega_{\mathrm{de}}}{\mathrm{d} z}\right],
\end{aligned}
$$




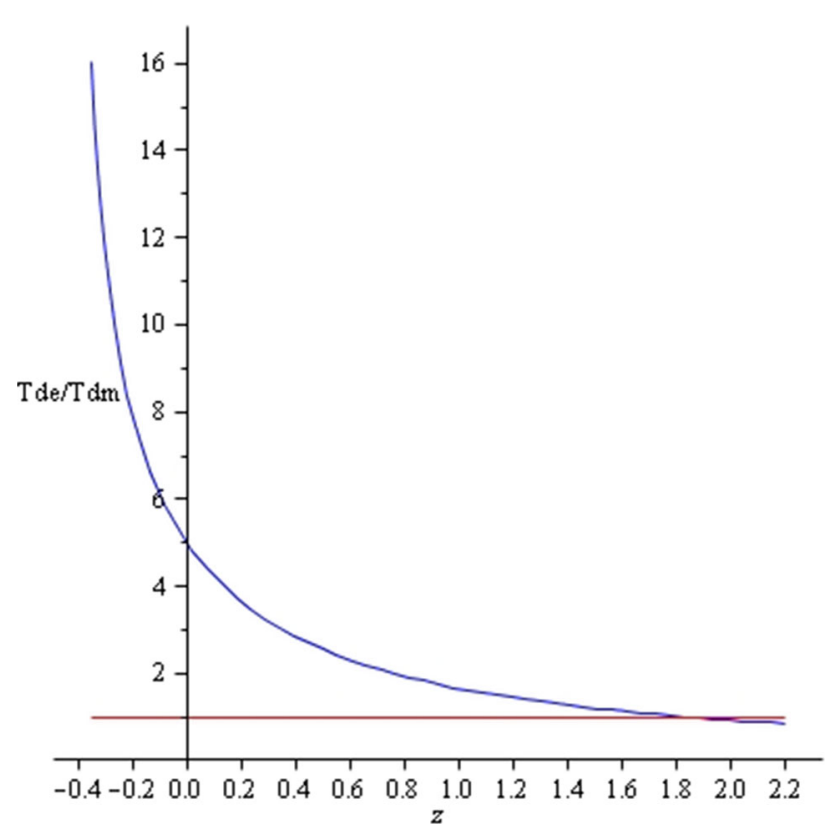

Fig. 2 We see that $T_{\mathrm{de}}>T_{\mathrm{dm}}$ in the range $-0.2<z<1.86$, i.e., when $Q>0$ and $T_{\mathrm{de}}<T_{\mathrm{dm}}$ for $z>1.86$, i.e., when $Q<0$, in accord to the second law. However, in the range $-0.39<z<-0.2$, when $Q<0$, the quotient $T_{\mathrm{de}} / T_{\mathrm{dm}}$ diverges

and $Q(z)$ can be written in the form

$\frac{Q}{9 H^{3}}(z)=-\frac{1}{3 \beta^{2} \sigma}(2 \alpha-3 \beta) \frac{(z-1.86)(z+0.2)}{(A+z)^{2}}$,

furthermore by using (9) and (10), the temperatures are given, respectively, by

$T_{\mathrm{de}}(z)=T_{\mathrm{de}}(0)(1+z)^{a}\left(1+\frac{z}{A}\right)^{b}$

and

$T_{\mathrm{dm}}(z)=T_{\mathrm{dm}}(0)(1+z)^{a}\left(1+\frac{z}{A}\right)^{b+1}\left(1+\frac{z}{B}\right)^{c}$,

where

$a=\left(\frac{2 \alpha-3 \beta}{\beta \sigma}\right)\left(\sigma+\frac{\omega(0)-\sigma A}{A-1}\right)$,

$b=-1-\left(\frac{2 \alpha-3 \beta}{\beta \sigma}\right)\left(\frac{\omega(0)-\sigma A}{A-1}\right)$,

$c=2 \alpha-3 \beta$;

$A=\frac{2+3 \beta \omega(0)}{3 \beta \sigma}, B=A-\left(\frac{2 \alpha-3 \beta}{3 \beta \sigma}\right)$,

where we have considered $\omega_{\mathrm{dm}}=0$ (dust). In Ref. [14] the best fit for the used parameters was $\left(\alpha, \beta, \omega_{\mathrm{de}}(0), \sigma\right)=$ $(0.73,0.38,-1.29,0.47)$ with $\chi^{2}=0.981$. All these parameters were estimated by using an adjustment with type I Supernova, Union 2 [29]. We note that the phantom case $\left(\omega_{\text {de }}(0)<-1\right)$ is required by this best fit. So, from them we have $Q(1.86)=Q(-0.2)=0$ and $a \approx 264.00$, $b \approx-264.15, c \approx 0.32 ; A \approx 0.98 B \approx 0.39$. For a better

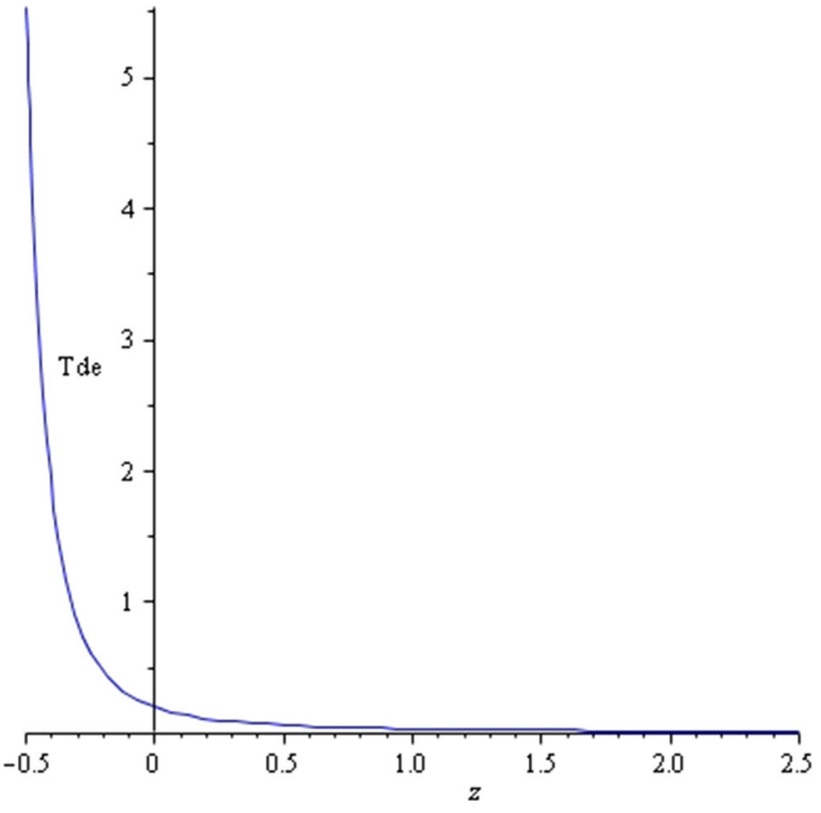

Fig. 3 We see that $T_{\mathrm{de}}$ always grows and the dark energy exhibits a negative heat capacity from $z \approx 1.86$ to $z \approx-0.2$ (dark energy is heated while energy is being delivered to dark matter). Out the indicated range, we have a positive heat capacity

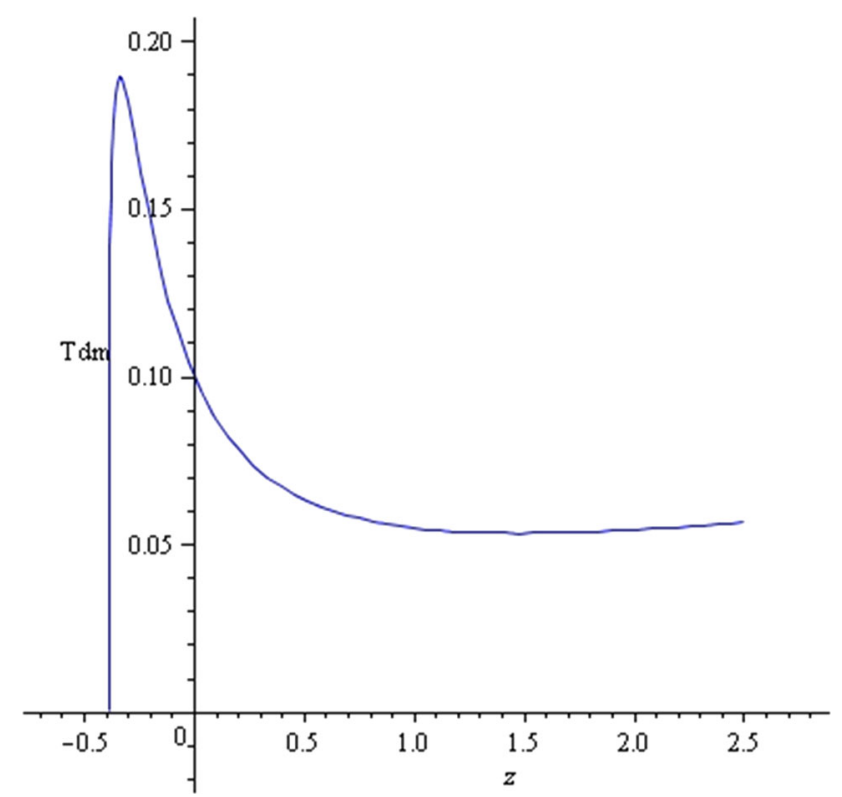

Fig. 4 We see that $T_{\mathrm{dm}}(z)$ grows toward $z=-0.39$. The dark matter heat capacity experiences a sign change, that is, from $z=\infty$ to $z=1.86$ we have a negative heat capacity (dark matter is heated while energy is being delivered to dark energy), from $z=1.86$ to $z=-0.2$ the heat capacity is positive, from $z=-0.2$ to $z=-0.33$ its heat capacity is negative (dark matter cools while receiving energy) and from $z=-0.33$ to $z=-0.38$ the heat capacity is positive

visualization we have chosen $T_{\mathrm{de}}(0) / T_{\mathrm{dm}}(0)>1$ (Figs. 2, 3 , and 4).

According to (28) and (29), we note that if $\omega_{\mathrm{de}}=$ const. and $\omega_{\mathrm{dm}}=0$ both temperatures are equal. This last situation 
does not appear to be consistent; is this a signal that $\omega_{\mathrm{de}} \neq$ const. through the evolution?

So, sign changes in $Q$ imply changes in the temperatures of dark energy and dark matter, as dictated by the second law. Additionally, we can visualize phase transitions, sign changes in its heat capacities, for both energy densities. Details can be found in the appendix.

Clearly, the observational data eventually will tell us if these changes will definitely occur. Today, the observational data is only just showing signals of the presence of $Q$, but as we stated above, it appears unnatural to think that cosmic fluids coexist and do not interact with each other. Additionally, we cannot say anything about values of $T_{\mathrm{de}}(0)$ and $T_{\mathrm{dm}}(0)$ : the question is whether future observations could elucidate or not this issue.

Finally, in the used parametrization for $\omega_{\mathrm{de}}(z)$, which makes sense for small values of $z$ [30,31], the behavior toward high $z$ is only formal. So, as can be seen from the figures, the essential scope lies in the region extending around today toward the near future where the emphasis has been put on the sign changes in the heat capacities of the energy densities and the behavior of the temperatures associated to them.

Roughly speaking, the sign change in the cosmic acceleration $(z \sim 0.6)$ is located "inside" the zone where $Q(z)>0$.

\section{Cosmological evolution}

We discuss briefly the behavior of the Hubble parameter, the coincidence parameter and the energy densities in the vicinity of the divergence of $T_{\mathrm{de}}(z)$, which occurs around $z \approx-0.4$, as shown in Fig. 3. According to (1), we write

$3 H^{2}=\rho_{\mathrm{de}}+\rho_{\mathrm{dm}}=\rho_{\mathrm{de}}(1+r)=\rho$,

where $r$ is the coincidence parameter given by the quotient $\rho_{\mathrm{dm}} / \rho_{\mathrm{de}}$ so that from $(25,26)$ we can write

$r=\frac{2(1-\alpha)+3 \beta\left(1+\omega_{\mathrm{de}}\right)}{2 \alpha-3 \beta}$,

and $\mathrm{d} r(z) / \mathrm{d} z=3 \beta \sigma /(2 \alpha-3 \beta)>0$, i.e., $\dot{r}(t)<0 \longleftrightarrow$ $2 \alpha-3 \beta>0$ (using the values for $\alpha$ and $\beta$ ).

By using (6), the energy density $\rho$ defined in (34) satisfies the conservation equation

$\dot{\rho}+3 H\left(1+\frac{\omega_{\mathrm{de}}}{1+r}\right) \rho=0$,

where we have considered $\omega_{\mathrm{dm}}=0$. So, from (34) and (35), it is straightforward to obtain the following solution for the Hubble parameter:
$H(z) / H(0)=C(1+z)^{\alpha / \beta}\left|1+\frac{2+3 \beta\left(\omega_{\mathrm{de}}(0)-\sigma\right)}{3 \beta \sigma(1+z)}\right|^{e}$,

where

$\begin{aligned} C & =\left|\frac{2+3 \beta \omega_{\mathrm{de}}(0)}{3 \beta \sigma}\right|^{-e} \\ e & =\frac{1}{\beta}\left(\frac{2 \alpha-3 \beta}{2+3 \beta\left(\omega_{\mathrm{de}}(0)-\sigma\right)}\right) .\end{aligned}$

According to the values for $\alpha, \beta$, and $\sigma$ used we have $e<0$. So, although $T_{\mathrm{de}}(z \longrightarrow-0.4)$ diverges, Fig. 3, $H(z \longrightarrow-0.4) \longrightarrow$ finite value, Fig. 5, and there is no future singularity in $H(z)$.

As regards the coincidence parameter, if we look at (37), we have

$r(z)>0 \Longrightarrow \omega_{\mathrm{de}}(z)>-\left[2\left(\frac{1-\alpha}{3 \beta}\right)+1\right]$,

and according to the used Ansatz for $\omega_{\mathrm{de}}(z)$, we have $r(z)>$ $0 \Longleftrightarrow z>-0.4$.

Finally, let us comment on the weak energy condition (WEC), which states that $\rho_{\mathrm{dm}}>0$ and $\rho_{\mathrm{de}}>0$, which implies according to (25)

$\rho_{\mathrm{de}}>0 \Longrightarrow z>-\frac{1}{\sigma}\left(\frac{2}{3 \beta}+\omega_{\mathrm{de}}(0)\right) \approx-1$,

and according to (26)

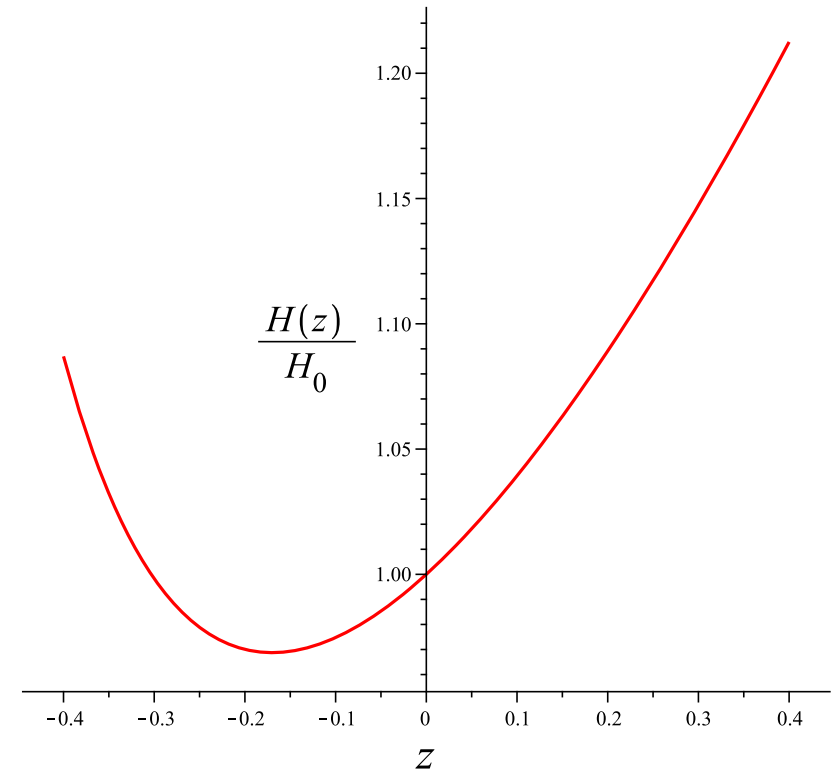

Fig. 5 The behavior of the Hubble parameter. There is no future divergence of $H(z)$ when $T_{\mathrm{de}}$ approximates $z=-0.4$ 


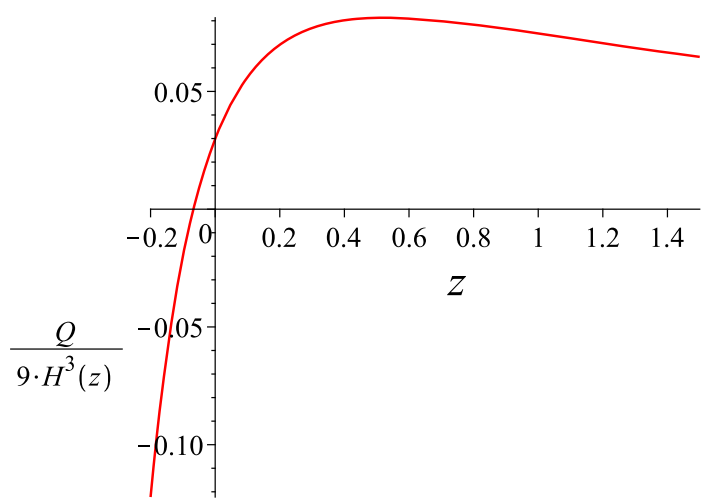

Fig. 6 The behavior of $Q$ and its sign change in the future

$\rho_{\mathrm{dm}}>0 \Longrightarrow z>-\frac{1}{\sigma}\left(\frac{2(1-\alpha)}{3 \beta}+1+\omega_{\mathrm{de}}(0)\right) \approx-0.4$,

and for $z>-0.4$ the WEC is satisfied and there are no future singularities in the energy densities.

We use now the CPL-parametrization [32] given by $\omega_{\mathrm{de}}(z)=\omega_{0}+\omega_{1} z(1+z)^{-1}$, where $\omega_{0}=\omega_{\mathrm{de}}(0)$ and $\omega_{1}=\omega_{\mathrm{de}}^{\prime}(0)$, in order to compare with the previous case.

At variance with the previous case, we see only one sign change in $Q(z)$ and this change occurs in the future, Fig. 6. However, as before, this fact also leads to sign changes in the heat capacities of both, dark energy and dark matter. See the appendix. So, there are no differences if we are thinking in the behavior of the heat capacities.

In this last section, we addresses the evolution of the temperatures. According to (9) and (10), we obtain, respectively,

$T_{\mathrm{de}}(z)=T_{\mathrm{de}}(0)(1+z)^{p_{1}}\left(1+\frac{a}{b} z\right)^{p_{2}} \exp \left(6 \omega_{1} \frac{z}{1+z}\right)$

(Fig. 7), and

$T_{\mathrm{dm}}(z)=T_{\mathrm{dm}}(0)(1+z)^{\sigma_{2}}\left(1+\frac{a}{b} z\right)^{\sigma_{1}}\left(1+\frac{A}{B} z\right)^{\sigma_{3}}$

(Fig. 8), where the parameters involved are given by

$$
\begin{gathered}
p_{1}=3\left[1+\frac{1}{\beta}(2 \alpha-3 \beta)-2\left(\omega_{0}+\omega_{1}\right)\right], \\
p_{2}=-3\left[1+\frac{2}{\beta}\left(\frac{2 \alpha-3 \beta}{2+3 \beta\left(\omega_{0}+\omega_{1}\right)}\right)\right],
\end{gathered}
$$

and

$\sigma_{i}=9(2 \alpha-3 \beta) \epsilon_{i}$

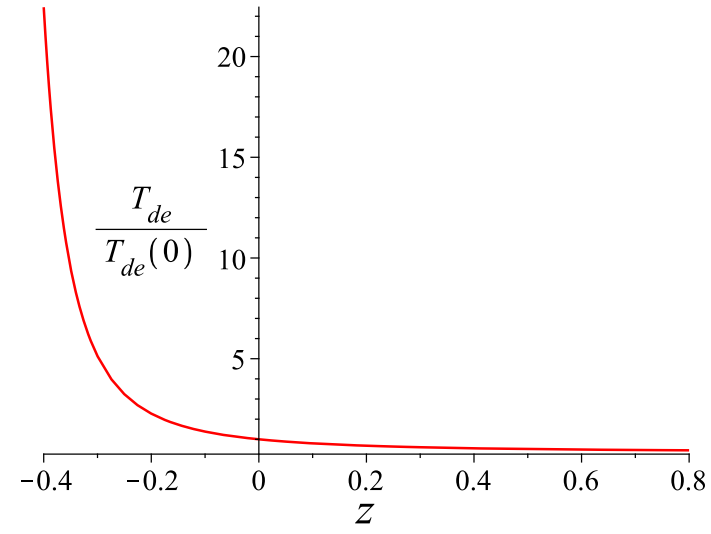

Fig. 7 We see that $T_{\text {de }}$ grows to the future and the dark energy exhibits a sign change in its heat capacity given the sign change experienced by $Q$

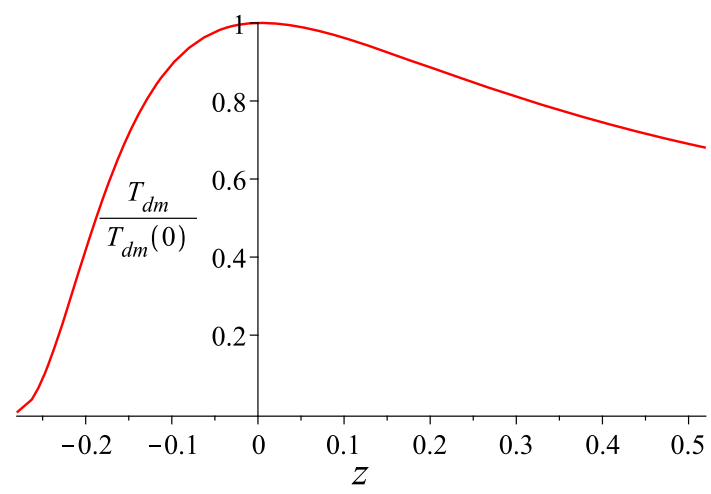

Fig. 8 We see that $T_{\mathrm{dm}}$ decreases to the future and the dark matter exhibits a sign change in its heat capacity given the sign change experienced by $Q$

where

$\epsilon_{1}=\frac{\omega_{0}+\omega_{1}}{a}+\frac{\beta \omega_{1}}{a A}\left(\frac{1}{B / A-b / a}\right)-\frac{1}{3 \beta}$,

$\epsilon_{2}=\frac{1}{3 \beta}$,

$\epsilon_{3}=-\frac{\beta \omega_{1}}{a A}\left(\frac{1}{B / A-b / a}\right)$,

and then

$$
\begin{aligned}
& a=2+3 \beta\left(\omega_{0}+\omega_{1}\right), \\
& b=2+3 \beta \omega_{0} ; \\
& A=2+3 \beta\left(\omega_{0}+\omega_{1}\right)-(2 \alpha-3 \beta), \\
& B=2+3 \beta \omega_{0}-(2 \alpha-3 \beta) .
\end{aligned}
$$

Finally, the Hubble parameter is given by

$$
H(z) / H(0)=(1+z)^{p_{3}}\left[1+\left(1+\frac{2}{3 \beta \omega_{0}}\right)^{-1} \frac{z}{1+z}\right]^{p_{4}}
$$




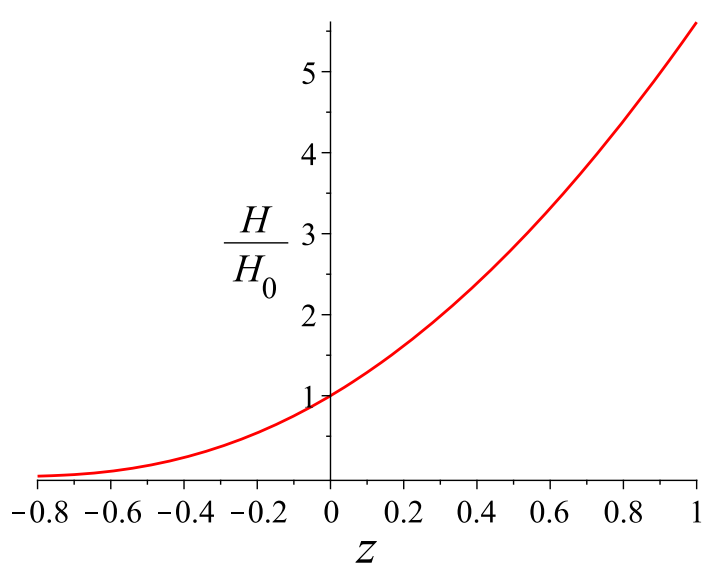

Fig. 9 The Hubble parameter

(Fig. 9), where

$$
\begin{gathered}
p_{3}=3\left[\frac{1+\alpha\left(\omega_{0}+\omega_{1}\right)}{2+3 \beta\left(\omega_{0}+\omega_{1}\right)}\right], \\
p_{4}=-\frac{1}{\beta}\left[\frac{2 \alpha-3 \beta}{2+3 \beta\left(\omega_{0}+\omega_{1}\right)}\right] .
\end{gathered}
$$

In order to plot Figs. 6, 7, 8, and 9 we have used the best fit for the parameters $\left(\alpha, \beta, \omega_{0}, \omega_{1}\right)=(0.86,0.23,-1.001$, $0.999)$ with $\chi^{2}=0.982$ [14]. All these parameters, as those used in the linear parametrization for $\omega_{\mathrm{de}}$, have been adjusted from type I Supernova, Union 2 [29]. In this case, the phantom case $\left(\omega_{0}<-1\right)$ is also required by this best fit.

We end this section by recalling that in the literature we can observe several parametrizations for dark energy (see [33], for instance). Regardless of this, the presence of interaction and the possibility of sign changes in the direction of energy transference between cosmological components, and mainly its consequences, are the key facts to inspect.

\section{Final remarks}

Consistently with the second law of thermodynamics, we have studied the behavior of the temperatures of two interacting fluids (dark energy and dark matter) and its relationship with the sign changes of $Q$ through the cosmic evolution. We have investigated the phase transitions (sign changes of its heat capacities) experienced for both, dark energy and dark matter. We have used a holographic model for the dark energy and, as usual, we have considered a pressureless fluid (dust) for dark matter.

It is generally believed that phase transitions have taken place in the early universe and its effects may well have been important for the late evolution. In the present work, phase transitions (seen here as sign change in the heat capacities of the fluids) are studied. Obviously phase transitions studied here are not like those experienced earlier, however, the pos- sibility of having future sign change in the heat capacities of the interacting cosmological components is in itself interesting to explore. So, negative heat capacities, as showed here, could reveal new physics beyond the usual thermodynamic limit. As we said before, the presence of interaction and possibility of sign change because of energy transfer between cosmological components can therefore be the key facts in order to inspect this issue. It is evident that we need a better theoretical approach, endorsed by the observational data, if we are thinking in the future evolution. Needless to say that the study realized before for the phantom case $(\omega<-1)$ may also be important.

We have used two modeling for $\omega_{\mathrm{de}}(z)$ and in both cases we have a sign change in $Q$. This fact is the highlight which leads to phase transitions and the behavior of the fluid temperatures. We emphasize that a sign change in $Q$ is relevant. The parametrization play an important role here, for example, in the linear case $\omega_{\mathrm{de}}(z)=\omega(0)+\sigma z$, we see two sign changes in $Q$, one in the past and the other in the future and for the CPL-model $\omega_{\mathrm{de}}(z)=\omega_{0}+\omega_{1} z(1+z)^{-1}$ we see only one sign change, in the future. Both $\omega_{\text {de }}(z)$-models represent a good description of the dark energy in the vicinity of $z=0$, time where we can better visualize the aforementioned changes.

Finally, and as we said at the beginning, it is natural to consider an interaction between cosmic fluids, in particular, between dark components which contribute in significant fractions to the contents of the universe. The presence of $Q$ appears to be compatible with the observations [34-36], such that this interaction could provide us an interesting insight to inspect the nature of the dark sectors, in particular, its thermodynamical nature. Additionally, if future observations were to detect sign changes in $Q$, very interesting consequences should appear in late cosmology.

Acknowledgments This work was supported from PUCV-VRIEA Grant No. 039.351/2016, Pontificia Universidad Católica de Valparaíso (S. L.) and DIUFRO Grant No. DI16-0066 of Dirección de Investigación y Desarrollo, Universidad de La Frontera (F. P.).

Open Access This article is distributed under the terms of the Creative Commons Attribution 4.0 International License (http://creativecomm ons.org/licenses/by/4.0/), which permits unrestricted use, distribution, and reproduction in any medium, provided you give appropriate credit to the original author(s) and the source, provide a link to the Creative Commons license, and indicate if changes were made. Funded by SCOAP ${ }^{3}$.

\section{Appendix: Heat capacities}

We write

$$
C=\frac{\Delta U}{\Delta T}
$$


and by putting $\Delta U=Q$ and recalling

$\dot{\rho}_{\mathrm{de}}+3 H\left(1+\omega_{\mathrm{de}}\right) \rho_{\mathrm{de}}=-Q$ and $\dot{\rho}_{\mathrm{dm}}+3 H \rho_{\mathrm{dm}}=Q$,

(6) we write

$$
\begin{gathered}
C_{\mathrm{de}}=\frac{-Q}{\Delta T_{\mathrm{de}}}<0, \text { if } Q>0 \text { and } \Delta T_{\mathrm{de}}>0, \\
C_{\mathrm{dm}}=\frac{+Q}{\Delta T_{\mathrm{dm}}}>0, \text { if } Q>0 \text { and } \Delta T_{\mathrm{dm}}>0 .
\end{gathered}
$$

Considering the temperatures, we see that $T_{\mathrm{de}}(z)$ always grows with $z\left(\Delta T_{\mathrm{de}}(z)>0\right)$ in the range $-0.2<z<1.86$; see Fig. 3. Then the dark energy heat capacity is

$C_{\mathrm{de}}(-0.2<z<1.86)=\frac{-Q}{\Delta T_{\mathrm{de}}}<0$,

and out of this range $C_{\mathrm{de}}=+Q / \Delta T_{\mathrm{de}}>0$.

We see also that $T_{\mathrm{dm}}$ grows with $z$ to -0.33 (maximum of $T_{\mathrm{dm}}$ ) and goes to zero when $z=-0.39$. In this range $\Delta T_{\mathrm{dm}}(z)>0$, but in the range $-0.39<z<-0.33$ we have $\Delta T_{\mathrm{dm}}(z)<0$. Then the dark matter heat capacity changes are

$C_{\mathrm{dm}}(1.86<z<\infty)=\frac{-Q}{\Delta T_{\mathrm{dm}}}<0$ and $\Delta T_{\mathrm{dm}}>0$,

$C_{\mathrm{dm}}(-0.2<z<1.86)=\frac{+Q}{\Delta T_{\mathrm{dm}}}>0$ and $\Delta T_{\mathrm{dm}}>0$,

$C_{\mathrm{dm}}(-0.2<z<-0.33)=\frac{-Q}{\Delta T_{\mathrm{dm}}}<0$ and $\Delta T_{\mathrm{dm}}>0$,

$C_{\mathrm{dm}}(-0.33<z<-0.39)=\frac{-Q}{\Delta T_{\mathrm{dm}}}>0$ and $\Delta T_{\mathrm{dm}}<0$.

By using the CPL-parametrization for dark energy, we write

$C_{\mathrm{de}}=\frac{-Q}{\Delta T_{\mathrm{de}}}$ and $\Delta T_{\mathrm{de}}>0 \longrightarrow\left\{\begin{array}{l}Q>0 \Longrightarrow C_{\mathrm{de}}<0, \\ Q<0 \Longrightarrow C_{\mathrm{de}}>0,\end{array}\right.$

and

$C_{\mathrm{dm}}=\frac{+Q}{\Delta T_{\mathrm{dm}}}$ and $\Delta T_{\mathrm{dm}}<0 \longrightarrow\left\{\begin{array}{l}Q>0 \Longrightarrow C_{\mathrm{dm}}<0, \\ Q<0 \Longrightarrow C_{\mathrm{dm}}>0 .\end{array}\right.$

So, as before, there are phase transitions.

\section{References}

1. E. Abdalla, E.G.M. Ferreira, J. Quintin, B. Wang. arXiv:1412.2777

2. B.Y. Pu, X.D. Xu, B. Wang, E. Abdalla. arXiv:1412.4091

3. V. Salvatelli, N. Said, M. Bruni, A. Melchiorri, D. Wands, Phys. Rev. Lett. 113(18), 181301 (2014)

4. T. Yang, Z.-K. Guo, R.-G. Cai, Phys. Rev. D 91, 123533 (2015)

5. S. Basilakos, M. Plionis, J. Sol á, Phys. Rev. D 80, 083511 (2009)

6. J.A.S. Lima, L.L. Graef, D. Pavón, S. Basilakos, JCAP 1410(10), 042 (2014)

7. P. Bull et al., arXiv:1512.05356

8. R. Maartens. arXiv:astro-ph/9609119

9. W. Zimdahl, Mon. Not. R. Astron. Soc. 288, 665 (1997)

10. N. Radicella, D. Pavón, Gen. Relativ. Gravit. 44, 685-702 (2012)

11. H. Zhang, X. Li, H. Noh, Phys. Lett. B 694, 177 (2010)

12. D. Bak, S.J. Rey, Class. Quantum Gravity 17, L83 (2000)

13. R.G. Cai, S.P. Kim, JHEP 0502, 050 (2005)

14. F. Arévalo, P. Cifuentes, S. Lepe, F. Peña, Astrophys. Space Sci. 352, 899 (2014)

15. D. Pavón, B. Wang, Gen. Relativ. Gravity 41, 1-5 (2009)

16. J.-M. Virey et al., Phys. Rev D 70, 121301 (2004)

17. I. Brevick, S. Nojiri, S.D. Odintsov, L. Vanzo, Phys. Rev D 70, 043520 (2004)

18. J.H. He, B. Wang, E. Abdalla, Phys. Lett. B 671, 139 (2009)

19. J.H. He, B. Wang, Y.P. Jing, JCAP 07, 030 (2009)

20. L.N. Granda, A. Oliveros, Phys. Lett. B 669, 275 (2008)

21. F. Yu, J.-L. Cui, J.-F. Zhang, X. Zhang. arXiv:1504.06067

22. C. Gao, X. Chen, Y.-G. Shen, Phys. Rev. D 79, 043511 (2009)

23. M. Li, Phys. Lett. B 603, 1 (2004)

24. R. Bousso, Rev. Mod. Phys. 74, 825-874 (2002)

25. Y. Hu, M. Li, N. Li, S. Wang. arXiv: 1501.06962

26. N. Kaloper, A. Padilla, Phys. Rev. Lett. 114(10), 101302 (2015)

27. S. Lepe, F. Peña, Astrophys. Space Sci. 350, 401 (2014)

28. S. Lepe, F. Peña, F. Torres, Phys. Rev D 91, 024023 (2015)

29. M. Kowalski et al., Astrophys. J. 686, 749 (2008)

30. J. Weller, A. Albrecht, Phys. Rev. D 65, 103512 (2002)

31. I. Maor, R. Brunstein, P. Steinhardt. arXiv:astro-ph/0007297

32. M. Chevallier, D. Polarski, Int. J. Mod. Phys. D 10, 213 (2001)

33. G. Pantazis, S. Nesseris, L. Perivolaropoulos. arXiv:1603.02164 [astro-ph.CO]

34. X.-D. Xu, E. Abdalla, The Universe 1(2), 29-38 (2013)

35. A.A. Costa, X.-D. Xu, B. Wang, E.G.M. Ferreira, E. Abdalla, Phys. Rev. D 89(10), 103531 (2014)

36. B. Wang, E. Abdalla, F. Atrio-Barandela, D. Pavón. arXiv:1603.08299 